\title{
A New Technique for Adapting SIP Protocol to Ad Hoc Networks: VNSIP (Virtual Network for SIP) Illustration and Evaluation of Performance
}

\author{
I. Mourtaji ${ }^{1}$, M. Bouhorma ${ }^{2}$, M. Benahmed ${ }^{3}$, A. Bouhdir ${ }^{4}$ \\ ${ }^{1234}$ LIST laboratory, FST of Tangier, Morroco \\ E-mail: 'imourtaji@gmail.com, ${ }^{2}$ bouhorma@gmail.com, ${ }^{3}$ med.benahmed@gmail.com, \\ ${ }^{4}$ hakim.anouar@gmail.com
}

\begin{abstract}
Ad Hoc Networks provide a real opportunity to design flexible networks, very simple to deploy. However they remain a particular computation environment, characterized by the deficiency of pre-existed and centralized infrastructure. In the other hand, SIP protocol, which knows a huge booming in internet networks, requires centralized entities, like proxy server, registrar server and location service; consequently SIP is not adapted to Ad Hoc networks. In this paper, we present and evaluate a new technique, which we have called Virtual Network for SIP (VNSIP) to fix the problem related to the constraints of SIP deployment in Ad Hoc network. The main idea of this technique is to create a virtual infrastructure, enabling SIP to proceed in a distributed architecture inside the Ad hoc Network.
\end{abstract}

Keywords: Ad hoc, SIP, Evaluation of performance

1 INTRODUCTION

Using this technique, we will be able to design a virtual sub-network, which will be used by SIP [1, $2,10]$ in a high mobility Ad Hoc network.

VNSIP allows decentralization of SIP proxies, specially registrar, proxy and location servers, by integrating those server functionalities in each MANET node. VNSIP node contains a supervisor module which we called Virtual Network Algorithm (VNA), and which is responsible of activating and deactivating server functionalities depending on the position of the node in the MANET [3].

This paper is organized as follows. In the first section we'll introduce VNSIP solution. In the second section we'll illustrate VNSIP design. Afterward in the next section, we'll present TCA solution. In the last section we will evaluate VNSIP performance using comparison with TCA approach. In the end, we will achieve this paper with a conclusion, with future works and perspectives.

\section{VNSIP SOLUTION PRESENTATION}

The presence of Proxy Server, Registrar Server and Location Service is a prerequisite for SIP operations. Thus it's necessary to decentralize those servers to enabling SIP integration in Ad Hoc netw The most evident solution, to fix this problem, is to include all SIP servers' functionalities in each node of the ad hoc network [4, 5]. However this solution consumes a lot of energy when broadcasting messages to find a node location [6], and generates many failure connections because of message collisions. Making this solution not optimal.

VNSIP solution tries to define a dynamic virtual network inside the MANET, to be exploited by nodes, to adequately choose which one will be in charge to execute SIP server tasks. VNA (Virtual Network Algorithm) will be the entity in charge to activate or deactivate SIP server functionalities in each MANET node. A VNSIP node (see Figure 1) consists of several entities, when interacting between them; they allow communication in MANET using SIP protocol. 


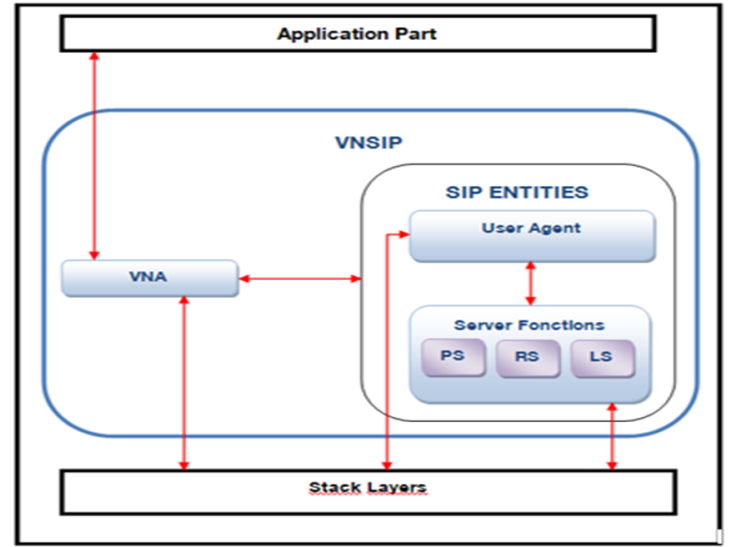

Fig. 1. VNSIP Node Architecture

\section{VNSIP DESIGN}

The first step is the construction of the Virtual Network (VN), which will be used by VNSIP to affect SIP server functionalities to the different nodes.

\subsection{Virtual Network Algorithm (VNA)}

VNA starts by the construction of neighbor tables. Thus each node of the MANET broadcasts a HELLO message [7]. When receiving this message for the first time, each node can populate its own neighbors table with its 1-hop neighbor's number information.

HELLO message is then sent for a second time, including the 1-hop neighbor's number information. When receiving the second HELLO message, each node upgrades its neighbors table with its 1-hop neighbor's number and 2-hop neighbors number information.

VNA defines a flag "VN_membership_flag" which shows if a node belongs to the $\mathrm{VN}$ or not. When executing VNA, the VN will include all nodes having VN_membership_flag=1. VNA is characteri-zed by two conditions (see Figure 2):

- Condition1: if a node doesn't belong to the $\mathrm{VN}$ and the number of its neighbors which belong to the $\mathrm{VN}$ is lower than the number of its neighbors which don't belong to the VN then the VN_membership_flag of this node is set to 1 .

- Condition 2: if a node belongs to the VN and the number of its neighbors which belong to the $\mathrm{VN}$ is higher than the number of its neighbors which don't belong to the VN then the VN_membership_flag of this node is set to 0 .

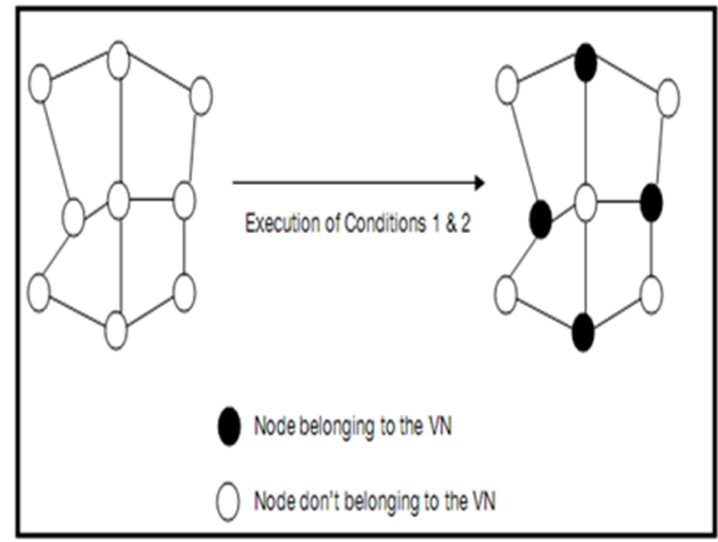

Fig. 2. Generation of nodes belonging to the VN

To complete the construction of a connected VN, we define Gateway nodes to ensure connections between all nodes belonging to the VN. Gateways are generated using the execution of the two rules below:

- Rule 1: if two nodes belonging to the $\mathrm{VN}$, and don't have a connection between them, and have a same neighbor node which doesn't belong to the $\mathrm{VN}$, then this node is considered as a Gateway.

- Rule 2: if two neighbor nodes, which don't belong to the $\mathrm{VN}$, and there neighbors are respectively belonging to the $\mathrm{VN}$, then those two nodes are considered as Gateways.

When finishing the selection of gateway nodes the construction of the $\mathrm{VN}$ is completed (see Figure 3).

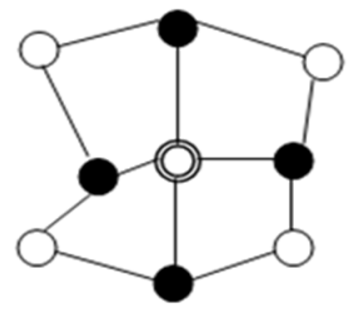

Node belonging to the $\mathrm{VN}$

Node don't belonging to the VN

Gateway

Fig. 3. VN construction 


\subsection{Interaction between VNA and SIP entities}

As it shown in Figure 3, when finishing the construction of the $\mathrm{VN}$, three kinds of nodes are defined:

- If a node belongs to the VN, then it plays SIP User Agent role and all its SIP server's functionalities (Proxy server (PS), Registrar (RG) and location service (LS)) are activated,

- If a node is a gateway, then it plays SIP User Agent role and PS role. RG and LS are deactivated. - If a node doesn't belong to the VN, then it plays only SIP User Agent role and its SIP server's functionalities are deactivated.

\subsection{Implementation of SIP Operations}

Registration:

When a node of the ad hoc network decides to registrar to the SIP network generated by VNSIP, two possibilities are treated:

- The node belongs to the VN: in this case, SIP servers for this node are activated. Thus its User Agent sends a Registrar message to its own RG, making a local registration (see Figure 4).

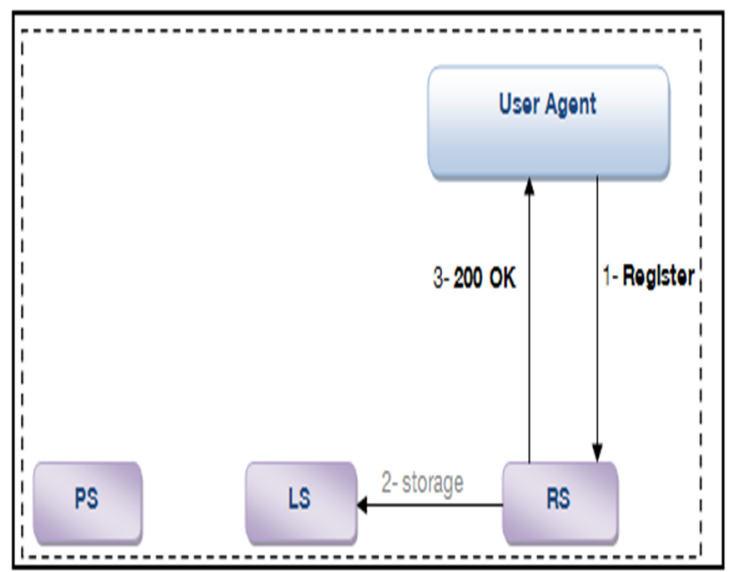

Fig. 4. Registration for a node belonging to the VN

The node doesn't belong to the VN: in this case, the User Agent of this node broadcasts a Register message to all its 1-hop neighbors, each neighbor register this node to its own RS if it belong to the VN (see Figure 5).

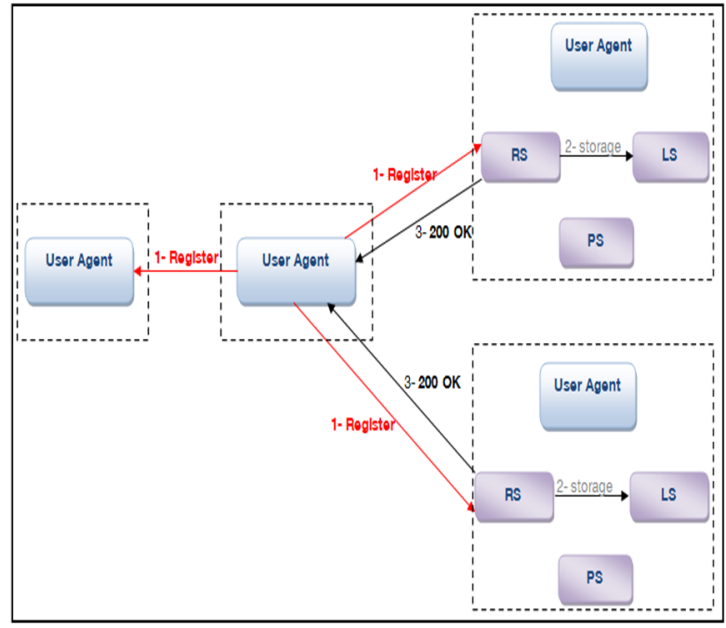

Fig. 5. Registration for a node don't belonging to the $V N$

\section{Session Initiation:}

When a node A decides to initiate a session with a node B. if SIP servers of A are activated then it sends INVITE message directly to its own PS (see Figure 6). Or if its SIP servers are deactivated, in this case, it broadcasts the INVITE to its 1-hop neighbors (see Figure 7).

When a PS receives the INVITE, it sends a request to its LS to verify in the data base if he knows the route to $\mathrm{B}$. if it does then the PS redirects the INVITE directly to B (see Figure 6). If not, it broadcast the INVITE to its 1-hop neighbors (see Figure 7).

When the User Agent of B receives the INVITE, its answers by a 200 OK message, which will follow the reverse path of the INVITE.

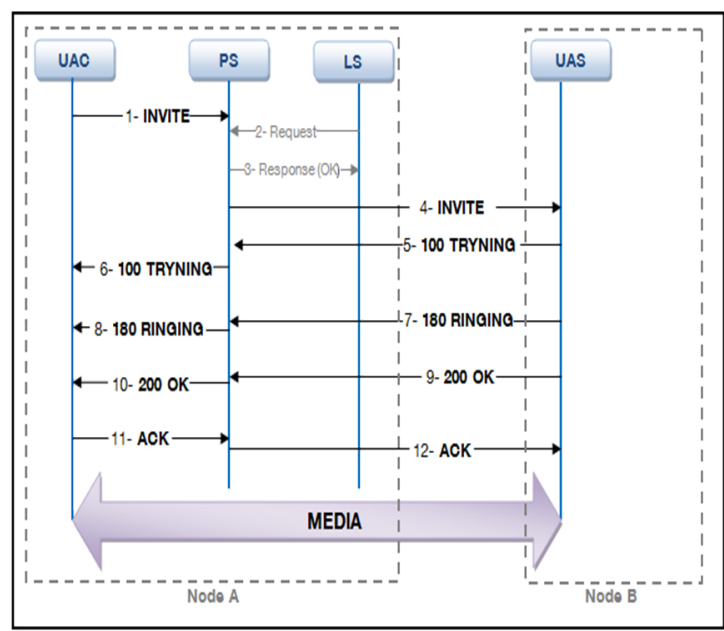

Fig. 6. PS of Node A knows location of Node B 


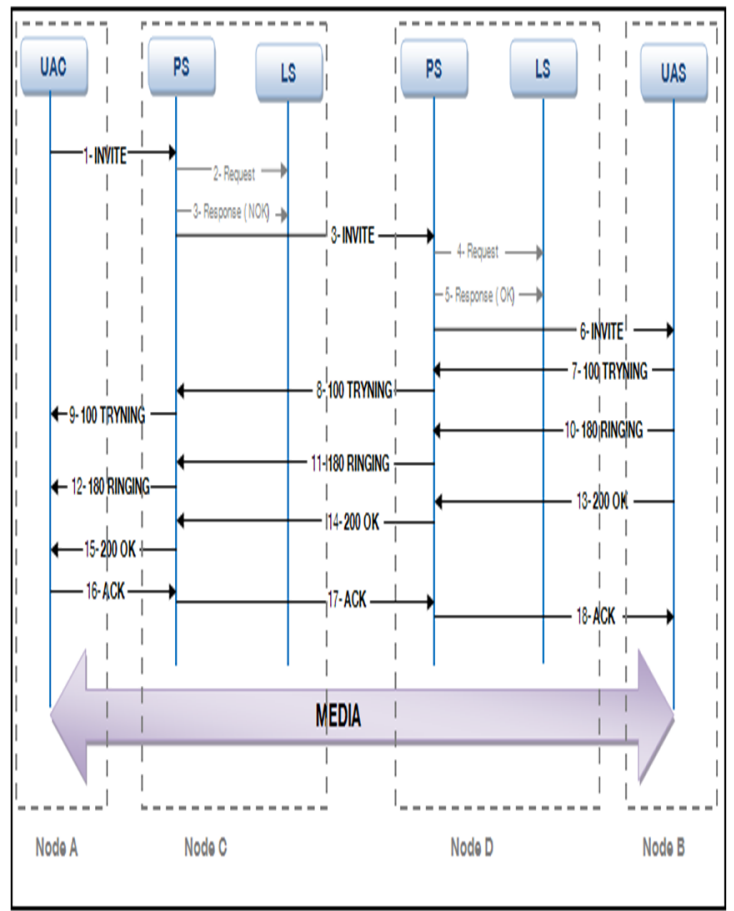

Fig. 7. PS of Node C doesn't know location of Node B

\section{TCA APPROACH}

In this chapter we will describe the design of TCA solution [9], to be compared with our solution VNSIP. TCA approach has been chosen because of its good results and because of its similarity with VNSIP.

\subsection{SIP terminal presentation in TCA}

For TCA approach, each node of the ad hoc network should be able to guarantee the functionalities below:

- $\quad$ SIP User Agent Client (UAC),

- SIP User Agent Server (UAS),

- SIP Proxy Server (PS),

- SIP Registrar (RG),

- SIP Location Server (LS),

- Topology construction

To achieve those functions, many models have been integrated in the same terminal; Figure 8 illustrates SIP node composition and interaction between those nodes.

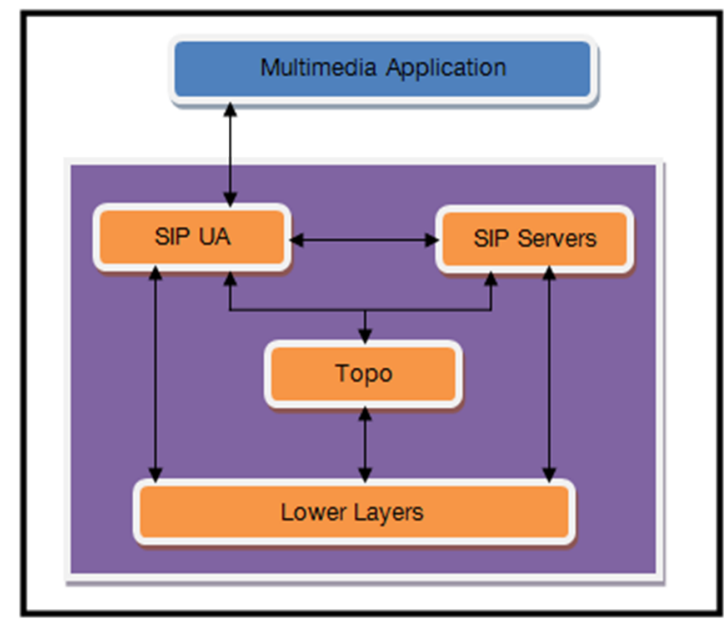

Fig. 8. TCA Node architecture

SIP UA module: realizes UAC and UAS functions, it initiates and responses to SIP requests. SIP Servers module: represents the different SIP servers (LS, RG and PS).

Topo module: constructs a cluster topology to be used by the different SIP modules.

\subsection{TCA Operating System}

TCA solution is essentially based on topology construction module, which is inspired by clustering approach. In fact, the topology construction algorithm chooses nodes to ensure cluster-head role. Nodes which not considered as cluster-heads will be considered as cluster members. Clusters will be related between them using gateway nodes (see Figure 9).

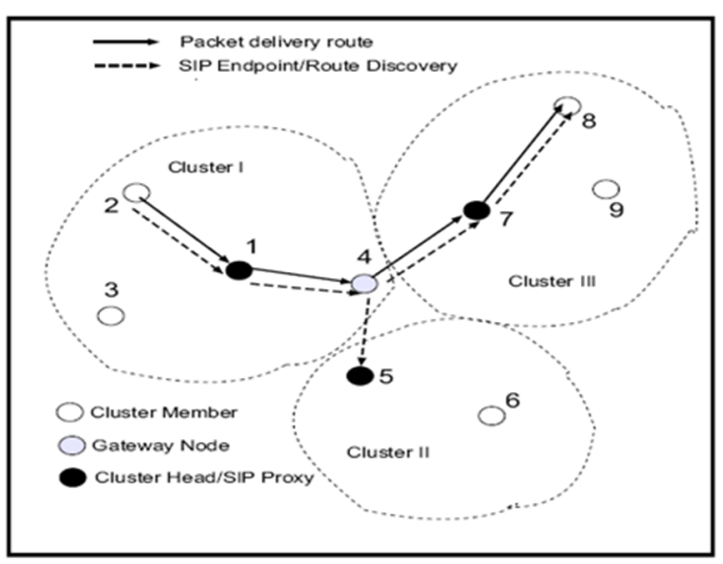

Fig. 9. Cluster construction 
When topology construction is achieved, the topo module assigns to each SIP module its role in the network. Each node is considered as a simple User Agent and according to its position in the network; additional tasks may be attributed to this node:

- A node which is considered as clusterhead will guarantee, in addition to UA role, PS, RG and LS role.

- A node which is cluster member and gateway node will guarantee, in addition to UA role, PS role.

- A node which is only considered as cluster member will guarantee a simple UA role

\section{SIMULATIONS AND EVALUATION OF PERFORMANCES}

\subsection{Configuration}

Simulations scenarios were achieved using the network simulator NS2 [8]. The simulation area was $1000 \mathrm{~m}$ by $1000 \mathrm{~m}$. The node number was between 10 and 50 nodes. The movement speed of nodes was between 0 and $18 \mathrm{~m} / \mathrm{s}$, and times of simulations were 180 seconds.

\subsection{Scenarios of simulations}

To define the difference between VNSIP behavior and TCA behavior, we achieved many types of simulations, and we analyzed the behaviors when node speeds and node numbers are increased.

\subsubsection{Session setting time}

The figure 10 illustrates session setting time, from TCA and VNSI, according to nodes mobility.

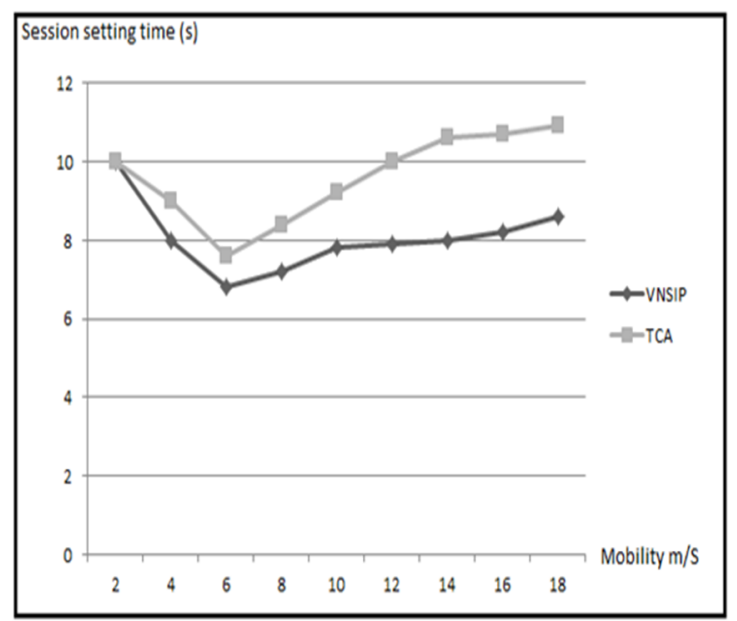

Fig. 10. Session setting time according to mobility
After analyzing the graph above, we can note that our approach guarantees a better time, than TCA solution, to establish sessions between nodes. We can justify this behavior by the fact that our solution VNSIP uses, to forward SIP messages, a higher number of nodes than TCA. And we can add that, replication mechanism used by VNSIP, allows performing many research on different SIP proxies on the same time, which helps to receive responses on shortest time.

The figure 11 illustrates session setting time, from TCA and VNSIP, according to the number of nodes

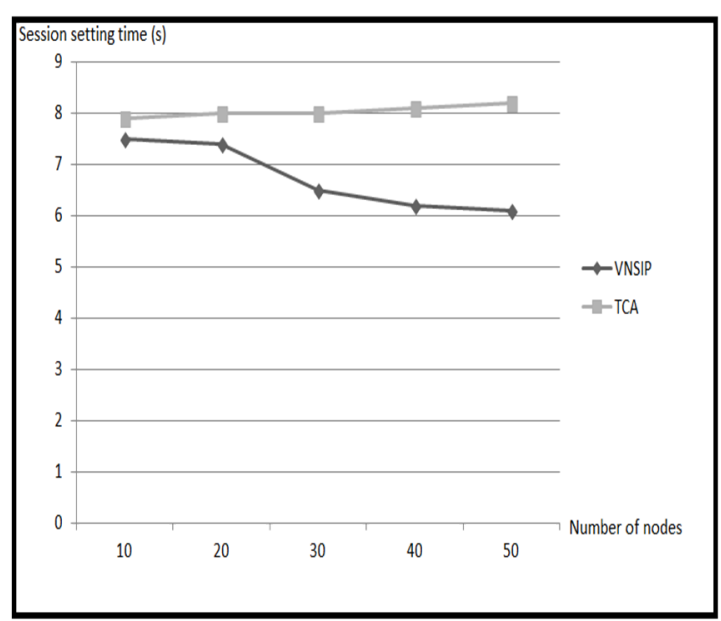

Fig. 11. Session setting time according to number of nodes

Comparing curves above, we observe that VNSIP gives better performance than TCA. In fact when the number of nodes, which are in charge to forward SIP messages, is increasing, it directly involves that session setting time decreased. Unlike TCA mechanism, which uses only one cluster-head to manage cluster members by forwarding SIP messages, generating largest queue and consequently the session setting time is increased.

\subsubsection{Failure rates}

The figure 12 illustrates the failure rates of session setting according to nodes mobility for TCA and VNSIP methods. 


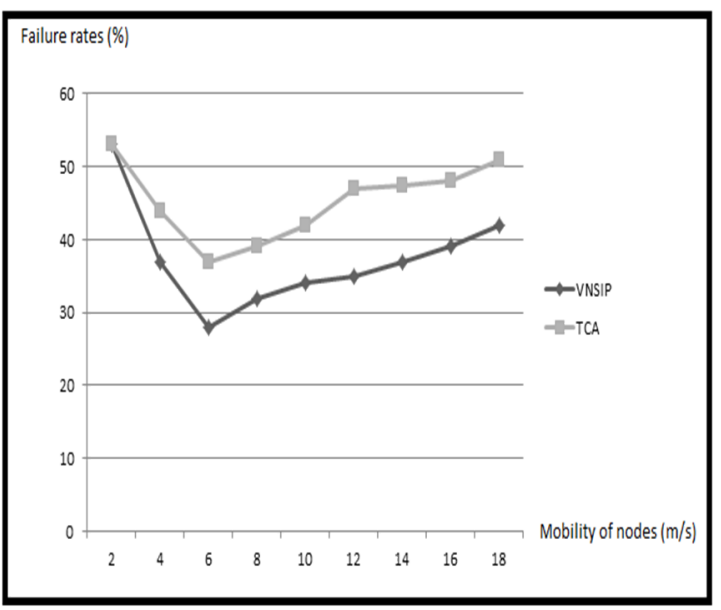

Fig. 12. Failure rates by mobility of nodes

When we compare failure rates to establish SIP sessions between both methods, we observe that VNSIP performs better results in terms of successful sessions setting. This result can be easily justified by the difference between VNSIP and TCA architectures. VNSIP uses replication mechanism, which allows forwarding SIP messages by several nodes, unlike TCA which uses only cluster-heads to forward SIP messages, this involves that there is only one registration for each node. Therefore TCA mechanism is very sensitive and vulnerable to cluster-heads moves.

\subsubsection{Bandwidth consumption}

The figure 13 illustrates bandwidth consumption when establishing SIP sessions according to number of nodes.

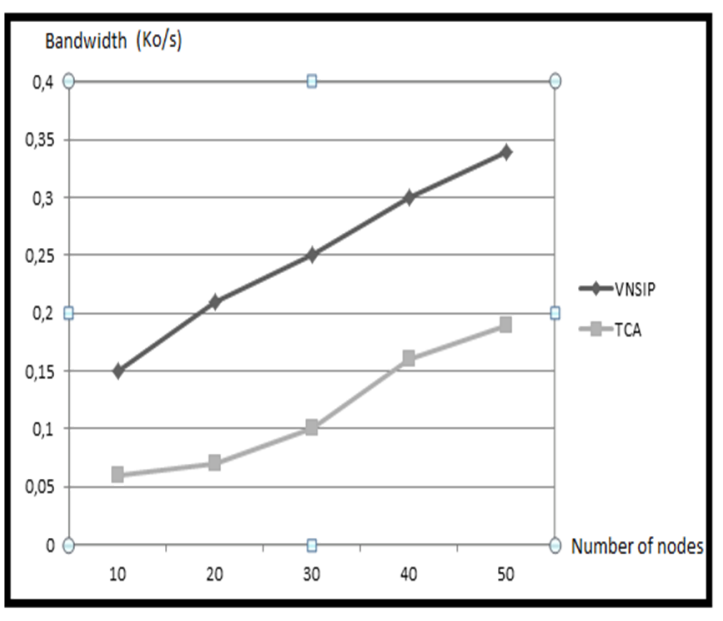

Fig. 13. Bandwidth consumption by number of nodes
When we compare both graphs, we note that VNSIP gives lower results than TCA; in fact consumption of bandwidth is higher and increases when number of nodes is rising. This behaviour can be warranted by the high number of message sent when applying our replication mechanism, which consists of achieving several registration and localization in the same time for the same node.

\section{CONCLUSION}

In this paper we have analyzed and evaluated the performance of a new approach for adapting SIP protocol to Ad Hoc Networks. To achieve this evaluation of performance, we have compared our solution to the TCA approach, which is consider as a solution giving a very interesting result to fix the problem of SIP adaptation to Ad Hoc Networks.

The simulation of both approaches has shown that our solution guarantees better results than TCA in terms of setting time and failure rates of SIP sessions. These good results were obtained thanks to VNA, the algorithm used to construct the VNSIP topology, and thanks to the mechanism of replication of SIP messages. On the other side, this mechanism has its own disadvantage, which involve that our solution proposes lower results than TCA in term of bandwidth consumption.

For our future works, we'll try to improve our solution to reduce the consumption of bandwidth. Actually we are designing a new algorithm, to guarantee a good MANET QoS.

\section{REFERENCES}

[1] J. Westcott et G. Lauer, 'Hierarchical routing for very large networks', Proc. IEEE MILCOM '84, pp 214-218, 21-24 October 1984.

[2] J. Rosenberg, H. Schulzrinne, G. Camarillo, A. Johnston, J. Peterson, R. Sparks, M. Handeley et E. Schooler, "SIP: Session Initiation Protocol", RFC 3261, Juin 2002.

[3] S. Corson, J. Macker, 'Mobile Ad hoc Networking (MANET): Routing Protocol Performance Issues and Evaluation Considerations', REC 2501, Janvier 1999.

[4] M. Jiang, J. Li and Y. C. Tay, "Cluster Based Routing Protocol (CBRP)," IETF Internet Draft draft-ietf-manet-cbrp-spec-01.txt, August 1999.

[5] C. R. Lin and M. Gerla, "Adaptive clustering for mobile, wireless networks," Journal on Selected Areas of Communication, Vol. 15, No. 7, 1997. 
[6] J. Rosenberg and H. Schulzrinne, "SIP: Locating SIP Servers", IETF RFC 3263, June 2002.

[7] C. Perkins, E. Belding-Royer, and S. Das “ Ad hoc On-Demand Distance Vector (AODV) Routing ”, IETF RFC 3561, July 2003.

[8] "The network simulator", available at http://www.isi.edu/nsnam/ns

[9] N. Banerjee, A. Acharya and S.K. Das, "Enabling SIP-Based Sessions in Ad Hoc Networks “, Winet, April 2006.

[10] A.B. Roach, "Session Initiation Protocol (SIP) - Specific Event Notification", RFC 3265, Juin 2002.

[11]P. Stuedi, M. Bihr, A. Remund et G. Alonso, « Siphoc : Efficient sip middleware for ad hoc networks " studi2007siphoc, LECTURE NOTES IN COMPUTER SCIENCE, 2007, Springer

[12] S. Leggio, J. Manner, A. Hulkkonen, et K. Raatikainen, "Session initiation protocol deployment in ad hoc networks: a decentralized approach", In 2nd International Workshop on Wireless Ad-hoc Networks (IWWAN), London, May, 2005. 